Journal of Mathematics and Informatics

Vol. 16, 2019, 77-85

ISSN: 2349-0632 (P), 2349-0640 (online)

Published 22 April 2019

www. researchmathsci.org

DOI: http://dx.doi.org/10.22457/jmi.140av16a7

Journal of

Mathematics and

Informatics

\title{
Evolutionary Game Research on the Supervision of Network Cars Based on the Network Platform under the Punishment Mechanism
}

\author{
Shi-jing Zhang \\ Basic Teaching Department \\ Yinxing Hospitality Management College of Chengdu University of Information \\ Technology, Chengdu, Sichuan, 611743, P.R. China. E-mail: zsj83@163.com
}

Received 1 April 2019; accepted 20 April 2019

\begin{abstract}
With the rapid development of the network car, the supervision problem of the network car platform has become increasingly prominent. In order to standardize the network car market and promote its healthy development, this paper studies the evolutionary game process of different strategic behaviors between the network car platform and the network car driver based on the penalty mechanism. The results show that the stable evolution strategy between the platform and the driver depends on the difference of benefits (or costs) between the penalty and the different strategies adopted by the two players. The higher the penalty, the faster the stable strategy will be achieved.
\end{abstract}

Keywords: Network car; Platform supervision; Punishment mechanism; Evolutionary game

\section{AMS Mathematics Subject Classification (2010): 91D10}

\section{Introduction}

The network car is a smart phone application software provided by Internet platform company for passengers. It is a way of travel for passengers to book vehicles to realize point-to-point transportation service [1]. As a representative of the sharing economy, the network car has improved the efficiency of the use of roads and vehicles, and has a significant impact on the convenience of people's lives. At the same time, because the network car platform has access to many private cars, it effectively reduces the car ownership and is beneficial to the sustainable development of the environment. Many scholars have done some research on the operation of the network car, such as Chen using Uber platform to analyze the impact of dynamic pricing on drivers in the platform [2], Becker studied the types of users participating in the web-based platform [3]. Wang Han-bin compared the operating modes of the three major network car companies and built a government-guided pricing model [4]. The pricing problem of taxis under the age of the network car should be studied smoothly and the suggestions for perfecting the taxi pricing system are given [5].With the rapid rise of the network car, a serious problem has gradually emerged, that is, the lack of effective supervision of this new operation mode. Several media reported that the network car drivers had bad behaviors, such as drinking, 


\section{Shi-jing Zhang}

driving fatigue and so on. In China, the supervision of the network car is still in the preliminary exploration stage. Many scholars have made relevant explorations on the management and supervision mode, quantity supervision and supervision path of the network car from the perspective of the government $[6,7,8,9]$. However, the existing research does not involve an interactive study of the regulatory strategies between the network platform and the network car.

In fact, it is difficult for both drivers and platforms to satisfy the assumption of complete rationality in the process of operation. The stable strategy of supervision should be formed in the long-term dynamic learning imitation [10]. Due to the short time of the network car, most scholars still stay in the exploration of the regulatory model for network car, and the dynamic evolution process of the network car supervision has not been studied. In this paper, based on the premise that both the platform and the driver are limited rationality, the evolutionary game method is used to study the evolutionary path of the platform to monitor the network car, which will provide some guidance for the healthy and sustainable development of the network car.

\section{Construction of evolutionary game model between network platform and network} car

(1) Model Assumptions and Symbolic Description

It is assumed that both the platform $N$ and the driver $D$ are limited rationality. The supervision strategy of the network car platform for the network car is "active supervision" and "negative supervision", while the strategy of the network car driver is "self-discipline" and "not self-discipline". The main symbolic descriptions are shown in Table 1.

Table 1: Symbol description

\begin{tabular}{|c|c|c|c|}
\hline symbol & Description & symbol & Description \\
\hline$C_{d 1}$ & $\begin{array}{c}\text { Cost of self-discipline for } \\
\text { network car driver }\end{array}$ & $C_{d 2}$ & $\begin{array}{c}\text { Cost of non-self-discipline for } \\
\text { network car driver }\end{array}$ \\
\hline$R_{d 1}$ & $\begin{array}{c}\text { Income of self-discipline for } \\
\text { network car drivers }\end{array}$ & $R_{d 2}$ & $\begin{array}{c}\text { Income of non-self-discipline } \\
\text { for network car drivers }\end{array}$ \\
\hline$C_{N 1}$ & $\begin{array}{c}\text { The cost of passive supervision } \\
\text { of the network platform }\end{array}$ & $C_{N 2}$ & $\begin{array}{c}\text { The cost of active supervision of } \\
\text { the network platform }\end{array}$ \\
\hline$p_{N}$ & $\begin{array}{c}\text { Fines for non-self-disciplined } \\
\text { acts are monitored by network } \\
\text { platform }\end{array}$ & $\lambda$ & $\begin{array}{c}\text { Probability of negative } \\
\text { supervision monitoring to non- } \\
\text { self-discipline behavior }\end{array}$ \\
\hline$w$ & $\begin{array}{c}\text { The loss of non-self-discipline } \\
\text { behavior for network platform }\end{array}$ & \multicolumn{2}{|c|}{} \\
\hline
\end{tabular}

According to the symbol of Table 1, the cost of self-discipline of the drivers should be higher than the cost of self-discipline, that is, $C_{d 1}>C_{d 2}$. If the drivers use cheating software or driving fatigue and other non-self-disciplined behavior, the benefits should be greater than the benefits of self-disciplined behavior, Therefore $R_{d 1}<R_{d 2}$. For the network car platform, if active supervision is adopted, the manpower and monitoring equipment will inevitably increase, so the cost of active supervision is higher than the cost of negative supervision, that is, $C_{N 1}<C_{N 2}$. According to the above assumptions, the income matrix of the game can be obtained as follows: 
Evolutionary Game Research on the Supervision of Network Cars Based on the Network Platform under the Punishment Mechanism

Table 2: Income matrix

\begin{tabular}{|c|c|c|c|}
\hline Game & \multicolumn{3}{|c|}{ Network car platform } \\
\hline \multirow{3}{*}{$\begin{array}{c}\text { Network } \\
\text { car } \\
\text { driver }\end{array}$} & & Active supervision & negative supervision \\
\hline & Self-discipline & $\left(R_{d 1}-C_{d 1},-C_{N 2}\right)$ & $\left(R_{d 1}-C_{d 1},-C_{N 1}\right)$ \\
\hline & $\begin{array}{l}\text { Non-self- } \\
\text { discipline }\end{array}$ & $\left(R_{d 2}-p_{N}-C_{d 2}, p_{N}-C_{N 2}-w\right)$ & $\left(R_{d 2}-\lambda p_{N}-C_{d 2}, \lambda p_{N}-C_{N 1}-w\right)$ \\
\hline
\end{tabular}

(2) Construction of Evolutionary Game Model

Assuming that the proportion of self-disciplined behavior is $x$, the proportion of nonself-disciplined behavior is $1-x$, the proportion of active supervision is $y$, and the proportion of passive supervision is $1-y$, the expected revenue and average expected revenue of the network car driver under self-disciplined behavior and non-self-disciplined behavior are recorded as $U_{x}, U_{1-x}$ and $\bar{U}_{d}$ respectively, the expressions are as follows:

$$
\begin{aligned}
& U_{x}=y\left(R_{d 1}-C_{d 1}\right)+(1-y)\left(R_{d 1}-C_{d 1}\right)=R_{d 1}-C_{d 1} \\
& U_{1-x}=y\left(R_{d 2}-P_{N}-C_{d 2}\right)+(1-y)\left(R_{d 2}-\lambda P_{N}-C_{d 2}\right)=R_{d 2}-C_{d 2}-(y+\lambda(1-y)) p_{N} \\
& \bar{U}_{d}=x U_{x}+(1-x) U_{1-x}
\end{aligned}
$$

The dynamic equation for the replication of $x$ is:

$$
\begin{aligned}
F(x) & =\frac{d x}{d t}=x\left(U_{x}-\bar{U}_{d}\right)=\left(x-x^{2}\right)\left(U_{x}-U_{1-x}\right) \\
& =\left(x-x^{2}\right)\left[\left(R_{d 1}-R_{d 2}\right)+\left(C_{d 2}-C_{d 1}\right)+\lambda p_{N}+(1-\lambda) p_{N} y\right]
\end{aligned}
$$

Similarly, the expected revenue and average expected revenue of the network platform under active supervision and negative supervision are recorded as $U_{y}, U_{1-y}$ and $\bar{U}_{N}$ respectively, the expressions are as follows:

$$
\begin{aligned}
& U_{y}=x\left(-C_{N 2}\right)+(1-x)\left(p_{N}-C_{N 2}-w\right)=(1-x) p_{N}-C_{N 2}-(1-x) w \\
& U_{1-y}=-x C_{N 1}+(1-x)\left(\lambda p_{N}-C_{N 1}-w\right)=-C_{N 1}+\lambda(1-x) p_{N}-(1-x) w \\
& \bar{U}_{N}=y U_{y}+(1-y) U_{1-y}
\end{aligned}
$$

The dynamic equation for the replication of $y$ is:

$$
\begin{aligned}
G(y) & =\frac{d y}{d t}=y\left(U_{y}-\bar{U}_{N}\right)=\left(y-y^{2}\right)\left(U_{y}-U_{1-y}\right) \\
& =\left(y-y^{2}\right)\left[C_{N 1}-C_{N 2}+(1-\lambda) p_{N}-(1-\lambda) p_{N} x\right]
\end{aligned}
$$

Then the replication dynamic equation of the dynamic system is:

$$
\left\{\begin{array}{l}
F(x)=\frac{d x}{d t}=\left(x-x^{2}\right)\left[\left(R_{d 1}-R_{d 2}\right)+\left(C_{d 2}-C_{d 1}\right)+\lambda p_{N}+(1-\lambda) p_{N} y\right] \\
G(y)=\frac{d y}{d t}=\left(y-y^{2}\right)\left[C_{N 1}-C_{N 2}+(1-\lambda) p_{N}-(1-\lambda) p_{N} x\right]
\end{array}\right.
$$


Shi-jing Zhang

3. Equilibrium analysis of evolutionary game between network car platform and network car

(1) Equilibrium Analysis

According to the stability theorem of differential equation and the properties of evolutionary stability strategy, the stability of the dynamic system is analyzed. The expression is as follows:

$$
\frac{d F(x)}{d x}=(1-2 x)\left(\left[\left(R_{d 1}-R_{d 2}\right)+\left(C_{d 2}-C_{d 1}\right)+\lambda p_{N}+(1-\lambda) p_{N} y\right]\right)
$$

Perform a stability analysis on the above expression, Let $\Delta R_{d}=R_{d 1}-R_{d 2}$, $\Delta C_{d}=C_{d 1}-C_{d 2}$, obviously $\Delta R_{d}$ indicates the difference between the benefits of selfdisciplined and non-self-disciplined behavior of the network car driver, and $\Delta R_{d}<0$. Similarly, $\Delta C_{d}$ indicates the cost difference between the self-discipline and non-selfdiscipline behavior of the network driver, and $\Delta C_{d}>0$. The equilibrium analysis of the strategy evolution of the network car driver is as follows:

1) If $0<\frac{1}{1-\lambda}\left(\frac{\Delta C_{d}-\Delta R_{d}}{p_{N}}-\lambda\right)<1$, it can be discussed in three cases:

(I) When $y=\frac{1}{1-\lambda}\left(\frac{\Delta C_{d}-\Delta R_{d}}{p_{N}}-\lambda\right), F(x)=0$ is established, so it is a stable strategy for all $x,(x \in[0,1])$;

(II) When $0<y<\frac{1}{1-\lambda}\left(\frac{\Delta C_{d}-\Delta R_{d}}{p_{N}}-\lambda\right)$, its stability strategy needs to satisfy $\frac{d F(x)}{d x}<0$, so $x_{1}^{*}=0$ is its stable evolution strategy. Under such circumstances, drivers always adopt non-self-discipline strategy;

(III) When $y>\frac{1}{1-\lambda}\left(\frac{\Delta C_{d}-\Delta R_{d}}{p_{N}}-\lambda\right), x_{1}^{*}=1$ is a stable evolutionary strategy. Under such circumstances, drivers always adopt a self-disciplined strategy.

2) If $\frac{1}{1-\lambda}\left(\frac{\Delta C_{d}-\Delta R_{d}}{p_{N}}-\lambda\right)<0, y>0>\frac{1}{1-\lambda}\left(\frac{\Delta C_{d}-\Delta R_{d}}{p_{N}}-\lambda\right), x_{1}^{*}=1$ is a stable evolutionary strategy, under such circumstances, drivers always adopt a selfdisciplined strategy.

3) If $\frac{1}{1-\lambda}\left(\frac{\Delta C_{d}-\Delta R_{d}}{p_{N}}-\lambda\right)>1, y<1<\frac{1}{1-\lambda}\left(\frac{\Delta C_{d}-\Delta R_{d}}{p_{N}}-\lambda\right), \quad x_{1}^{*}=0$ is a stable evolutionary strategy, under such circumstances, drivers always adopt a non-selfdisciplined strategy.

In the same way, the evolution equilibrium analysis of the strategy of the network car platform is carried out. The derivative of $G(y)$ can be obtained as follows: 
Evolutionary Game Research on the Supervision of Network Cars Based on the Network Platform under the Punishment Mechanism

$$
\frac{d G(y)}{d y}=(1-2 y)\left[C_{N 1}-C_{N 2}+(1-\lambda) p_{N}-(1-\lambda) p_{N} x\right]
$$

The stability strategy requires $\frac{d G(y)}{d y}<0$ to be satisfied, hypothesis $\Delta C_{N}=C_{N 1}-C_{N 2}$ indicates the degree of difference between the cost of negative supervision and active supervision of the network car platform. According to the assumption, it is obvious that $\Delta C_{N}<0$ is established, so $\frac{\Delta C_{N}}{(1-\lambda) p_{N}}+1<1$ must be established. The equilibrium evolution strategy is as follows:

1) If $\frac{\Delta C_{N}}{(1-\lambda) p_{N}}+1>0$, it can be discussed in three cases:

(I) When $x=\frac{\Delta C_{N}}{(1-\lambda) p_{N}}+1$, it is a stable strategy for all $y,(x \in[0,1])$;

(II) When $x>\frac{\Delta C_{N}}{(1-\lambda) p_{N}}+1, y_{1}^{*}=0$ is a stable evolutionary strategy. Under such circumstances, the network car platform always adopts a negative supervision strategy;

(III) When $x<\frac{\Delta C_{N}}{(1-\lambda) p_{N}}+1, \quad y_{2}{ }^{*}=1$ is a stable evolutionary strategy. Under such circumstances, the network car platform always adopts an active supervision strategy.

2) If $\frac{\Delta C_{N}}{(1-\lambda) p_{N}}+1<0$, there will always be $x>0>\frac{\Delta C_{N}}{(1-\lambda) p_{N}}+1, y_{1}^{*}=0$ is still a stable evolutionary strategy. Under such circumstances, the network car platform always adopts a negative supervision strategy.

(2) Numerical simulation analysis

In order to visually observe the evolution process of the network car platform and the network car driver strategy under different parameter settings, this paper will carry out numerical simulation. Suppose that at the beginning, the probability that the network car platform chooses to actively supervise is low, $y(0)=0.3$, and the strategy of the network car driver adopting self-discipline is also low, $x(0)=0.3$. Under the negative supervision, the possibility that the network car platform can detect the bad behavior of the network car is $\lambda=0.4$, the penalty is $p_{N}=50$, and $y_{0}=\frac{1}{1-\lambda}\left(\frac{\Delta C_{d}-\Delta R_{d}}{p_{N}}-\lambda\right)$ is taken as $-0.2,0.4,1.2, x_{0}=\frac{\Delta C_{N}}{(1-\lambda) p_{N}}+1$ is taken as $-0.5,0.1$, respectively. Numerical simulation analysis is carried out. The detailed results are shown in the following figure: 
Shi-jing Zhang

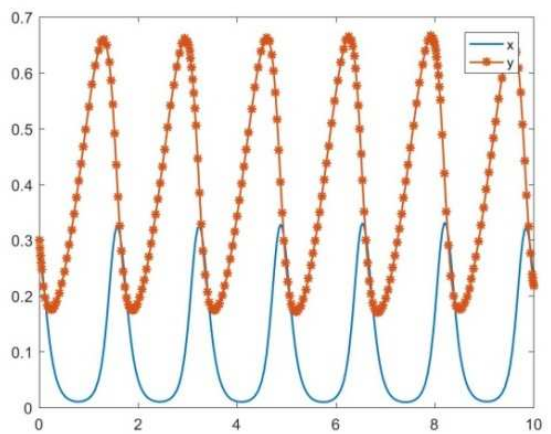

Figure 1: $(a) \quad x_{\circ}=0.1, y_{\circ}=0.4, P_{v}=50$

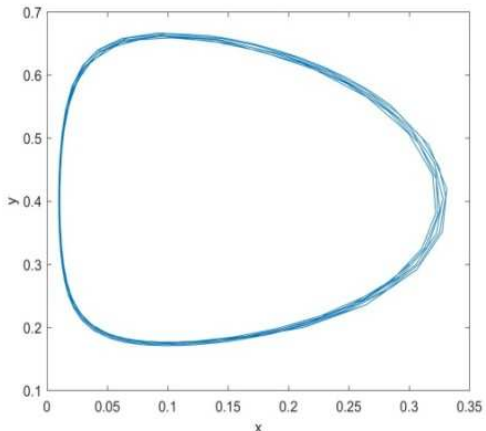

Figure 1: (b) $x_{0}=0.1, y_{0}=0.4, P_{v}=50$

According to Fig. 1(a)-(b), it can be seen that when $0<x_{0}<1,0<y_{0}<1$, there is $\left|\Delta C_{N}\right|<(1-\lambda) p_{N}$ and $\lambda p_{N}<\Delta C_{d}-\Delta R_{d}<p_{N}$. When the network platform adopts different regulatory strategies, the cost difference is lower than the penalty, and the profit-cost difference caused by the different behaviors of the drivers is within a certain proportion of the penalty. There is no stable evolutionary game strategy between the two sides of the game, and the evolutionary games of the two players are dependent on each other and change periodically. There is no stable point.

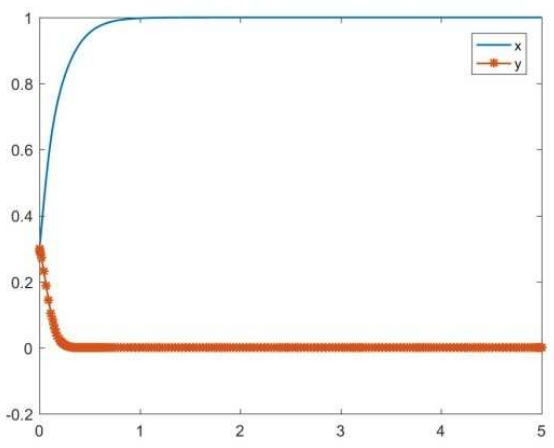

Figure 2: $(a) \quad x_{0}=0.1, y_{0}=-0.2, P_{v}=50$

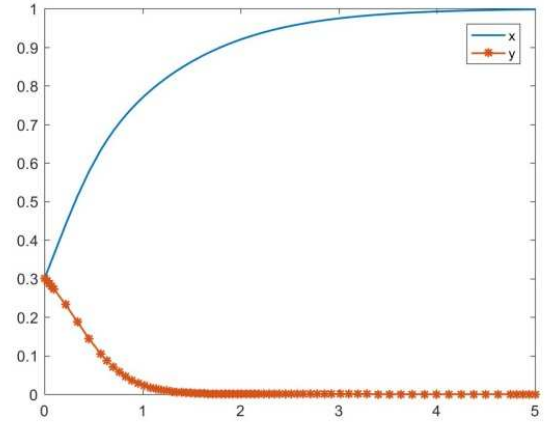

Figure 2: (b) $x_{0}=0.1, y_{\mathrm{o}}=-0.2, P_{\mathrm{s}}=10$

According to Fig. 2(a)-(b), it can be seen that when $0<x_{0}<1,0<y_{0}<1$, there is $\left|\Delta C_{N}\right|<(1-\lambda) p_{N}$ and $\Delta C_{d}-\Delta R_{d}<\lambda p_{N}$. In the case of a long-term dynamic game, when the sum of the difference in the revenue cost of the network car driver is lower than the expected value of the penalty, the penalty is higher. At this time, the driver always chooses the self-discipline behavior. The strategy of negative supervision will be adopted. As the penalty money increases, the speed of reaching the stability strategy increases rapidly. At this time, the impact of the penalty on the strategy of both sides of the game is very significant. 
Evolutionary Game Research on the Supervision of Network Cars Based on the Network Platform under the Punishment Mechanism

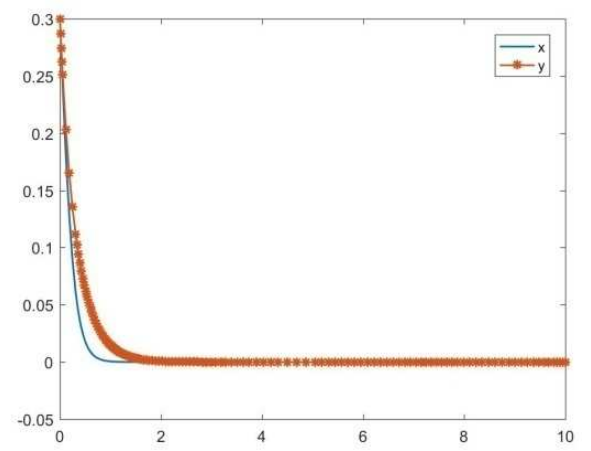

Figure 3: $x_{0}=-0.5, y_{0}=1.2, P_{\mathrm{N}}=10$

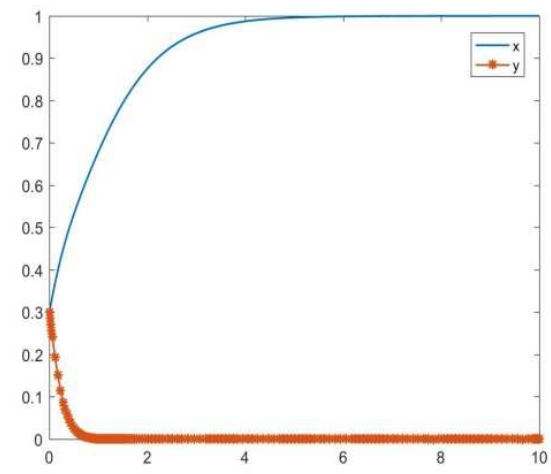

Figure 4: $x_{0}=-0.5, y_{0}=-0.2, P_{N}=10$

According to Fig. 3-4, it can be seen that when $x_{0}<0$, there is $\left|\Delta C_{N}\right|>(1-\lambda) p_{N}$. When the cost difference between active supervision and passive supervision is higher than the penalty, the penalty is weaker for the platform. Whether the difference between the profit and cost of different behaviors is lower or higher than the penalty, the platform always adopts passive supervision, but the driver will choose different behavior according to the size of the punishment.

Under the above circumstances, if there is a stable evolution strategy in the network car platform, it is a strategy of selecting negative supervision. But when $0<x_{0}<1, y_{0}>1$, there is $\left|\Delta C_{N}\right|<(1-\lambda) p_{N}$ and $\Delta C_{d}-\Delta R_{d}>p_{N}$. When the punishment is low for the drivers, the drivers always try to adopt non-self-disciplined behavior. In order to cope with the behavior of the drivers, the platform will take the initiative to supervise. The specific simulation results are shown in Fig. 5-6.

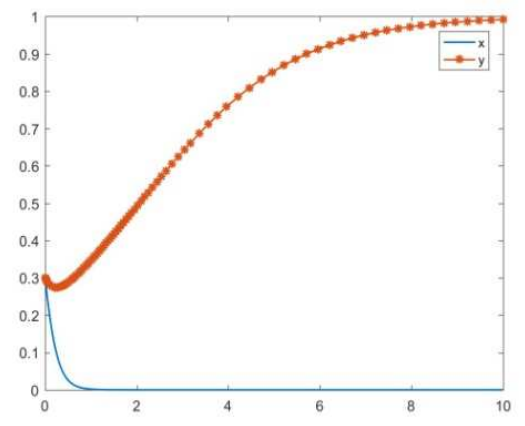

Figure 5: $x_{0}=0.1, y_{0}=1.2, P_{v}=10$

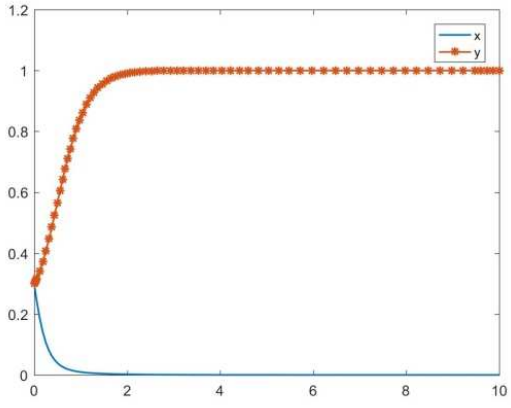

Figure 6: $x_{0}=0.5, y_{0}=1.2, P_{N}=10$

Fig. 5-6 also shows that with the increase of $x_{0}$, the faster it converges to the stabilization strategy, while the increase of $x_{0}$ requires the increase of $\frac{\Delta C_{N}}{(1-\lambda) p_{N}}$. 


\section{Shi-jing Zhang}

Because of $\Delta C_{N}<0$, it can be seen that the higher the penalty, the faster the convergence to the stabilization strategy.

\section{Conclusion}

As a new business model, the lack of management makes it very difficult to supervise the drivers. Based on this background, this paper assumes that both drivers and platforms are bounded rationality. Through the establishment of evolutionary game model, it proposes how to establish a penalty mechanism to achieve the supervision of drivers. And get the following conclusions:

(1) As for the platform and driver of the online reservation vehicle, the degree of punishment directly affects the choice of their strategy and behavior, and the stronger the punishment, the faster it achieves the stable strategy.

(2) When the network platform adopts different regulatory strategies, the cost difference is lower than the penalty, and the profit-cost difference caused by the different behaviors of the drivers is within a certain proportion of the penalty, the two sides of the game can not achieve the stability strategy, but depend on each other's choice. The stabilization strategies of both players depend mainly on the degree of difference between penalty money and different tactical actions taken by one of the players.

To sum up, whether for the drivers or the platforms, the introduction of appropriate punishment mechanism can effectively promote both sides of the game to achieve a stable strategy. Especially from the perspective of the platforms, we should strengthen the supervision, constantly improve the supervision system and increase its punishment, so as to promote the self-discipline behavior of the network car drivers in the economic activities, in order to achieve a healthy and sustainable development of the network car market.

\section{REFERENCES}

1. W.Jing, Regulatory dilemma and solution of China's network car, Administrative Law Review, 96(2) (2016) 49-59.

2. M.K.Chen, Dynamic Pricing in a Labor Market: Surge Pricing and Flexible Work on the Uber Platform, ACM Conference on Economics and Computation, ACM, (2016) 455-455.

3. H.Becker, F.Ciari and K.W.Axhausen, Comparing car-sharing schemes in Switzerland: User groups and usage patterns, Transportation Research Part A Policy \& Practice, 97 (2017) 17-29.

4. W.Hanbin and Y.Shuai, Research on the pricing model of the network car, Prices Monthly, 10 (2016) 32-36.

5. Y.Chang, Research on pricing mechanism of taxi freight rate in the age of network cars, Price Theory and Practice, 10 (2016) 98-101.

6. X.Mingyue and L.Hengke, Reform and improvement of legal supervision of local taxi market under the background of network car, Social Sciences in Guangdong, 5 (2016)249-256.

7. H.Denghua, The operation mode of the network car under the "Quadruple 
Evolutionary Game Research on the Supervision of Network Cars Based on the Network Platform under the Punishment Mechanism

Agreement" and its supervision path, Law Science Magazine, 37(12) (2016) 68-77.

8. Z.Yikai, Research on the new policy of online vehicle restriction, China Industrial Economics, 8 (2017) 81-99.

9. X.Zhiyuan, Research on the control of the number of vehicles in network appointment, Theory and Reform, 6 (2016) 108-113.

10. L.Wei, X.Liqiu and W.Yilei, Evolutionary game analysis of internet financial platform behavior and supervision strategy under dynamic penalty mechanism, System Engineering Theory and Practice, 37(5) (2017) 1113-1122. 